\title{
A Comparison between the 4DVAR and the Ensemble Kalman Filter Techniques for Radar Data Assimilation
}

\author{
A. CAya, J. Sun, And C. SNyder \\ National Center for Atmospheric Research,* Boulder, Colorado
}

(Manuscript received 16 March 2004, in final form 21 March 2005)

\begin{abstract}
A four-dimensional variational data assimilation (4DVAR) algorithm is compared to an ensemble Kalman filter (EnKF) for the assimilation of radar data at the convective scale. Using a cloud-resolving model, simulated, imperfect radar observations of a supercell storm are assimilated under the assumption of a perfect forecast model. Overall, both assimilation schemes perform well and are able to recover the supercell with comparable accuracy, given radial-velocity and reflectivity observations where rain was present. 4DVAR produces generally better analyses than the EnKF given observations limited to a period of $10 \mathrm{~min}$ (or three volume scans), particularly for the wind components. In contrast, the EnKF typically produces better analyses than 4DVAR after several assimilation cycles, especially for model variables not functionally related to the observations. The advantages of the EnKF in later cycles arise at least in part from the fact that the 4DVAR scheme implemented here does not use a forecast from a previous cycle as background or evolve its error covariance. Possible reasons for the initial advantage of 4DVAR are deficiencies in the initial ensemble used by the EnKF, the temporal smoothness constraint used in 4DVAR, and nonlinearities in the evolution of forecast errors over the assimilation window.
\end{abstract}

\section{Introduction}

Data assimilation at the convective scale poses numerous challenges. Doppler radars provide sufficient temporal and spatial coverage to resolve moist convection, yet they observe only a single component of the velocity and reflectivity, which is a complicated function of the microphysical species. Because balances like geostrophy between the wind and mass fields are weak or absent, the assimilation scheme must use other dynamical information in order to initialize unobserved variables such as temperature. Two methods that incorporate such information are four-dimensional variational data assimilation (4DVAR; Lewis and Derber 1985; Le Dimet and Talagrand 1986; Courtier and Talagrand 1987; Klinker et al. 2000) and the ensemble Kalman filter (EnKF; Evensen 1994; Houtekamer and

\footnotetext{
* The National Center for Atmospheric Research is sponsored by the National Science Foundation.
}

Corresponding author address: Alain Caya, NCAR, P.O. Box 3000, Boulder, CO 80307-3000.

E-mail: caya@ucar.edu
Mitchell 1998). In this paper, we compare 4DVAR and the EnKF in the context of convective-scale assimilation.

Both 4DVAR and the EnKF have shown promise at convective scales. Using a limited domain and observations of a single convective cell, Sun and Crook (1997, 1998) demonstrated a 4DVAR scheme for both simulated and real radar observations. Similar techniques have also been applied to the assimilation of clear-air radar returns in real time (Sun and Crook 2001; Crook and Sun 2002). More recently, the EnKF has also been tested successfully for the assimilation of simulated radar observations of single convective cells (Snyder and Zhang 2003; Zhang et al. 2004) and for the assimilation of real radar observations of a supercell (Dowell et al. 2004).

Previous comparisons of 4DVAR and the EnKF are limited largely to idealized, low-order systems. In such systems, the performance of the EnKF has compared favorably to that of 4DVAR (Hansen and Smith 2001; Anderson 2001; Hansen 2002). The EnKF has also been compared with a weak-constraint 4DVAR approach for the assimilation of microwave brightness temperature into a land surface model (Reichle et al. 2002). The EnKF is yet to be evaluated against 4DVAR for highdimensional systems or for realistic atmospheric flows. 
Aside from direct comparisons of the schemes, estimation theory also provides insights into the relation of 4DVAR and the EnKF. In the theoretical context of a linear forecast model, linear observational operator, and Gaussian statistics, 4DVAR and the Kalman filter give identical results at the end of the assimilation when model error is neglected (Lorenc 1986, section 4h). Furthermore, under these same linear, Gaussian assumptions and for sufficiently large ensembles, the EnKF agrees with the Kalman filter. Inherent nonlinearities at the convective scale (and the resulting non-Gaussian distributions) are thus a potential cause of differences between 4DVAR and the EnKF. In the case that background and observation errors remain Gaussian but model dynamics are nonlinear, 4DVAR yields the maximum likelihood estimate, that is, the conditional mode of the posterior probability density function (pdf) (Cohn 1997, section 3.3). It is not clear in general how much the conditional mode improves on the EnKF analysis, which is linear and depends only on the second moment of the prior pdf, though simple examples have shown the importance of non-Gaussian effects when nonlinearity is sufficiently strong (Anderson and Anderson 1999; Bengtsson et al. 2003).

In most geophysical problems, however, the dimension of the state is so large that practical schemes are inevitably a compromise between computational necessities and theoretically optimal approaches. For example, the EnKF suffers sampling errors from limited ensemble sizes, and, since the matrices are too large to manipulate easily, 4DVAR must use tractable, and typically idealized, models of the background error covariances. Minimization of the 4DVAR cost function is also often difficult without the addition of constraints to improve the conditioning of the problem and speed the minimization. Thus, differences in 4DVAR and EnKF performance are expected from purely practical considerations, in addition to any effects of nonlinearity.

Certain aspects of convective-scale assimilation make the comparison of 4DVAR and the EnKF particularly interesting. First, convective dynamics are distinctly nonlinear. The microphysical processes that drive the convection include both nonlinear thresholds (such as the onset of condensation once a parcel reaches saturation) and transformations among species that are strongly nonlinear functions of their concentrations. Second, the background error covariances are especially crucial for the EnKF because of the need to spread information from the observations to the unobserved components of velocity and the temperature field. At the same time, the balances between the velocity and mass field that hold at synoptic and larger scales typically do not pertain at convective scales, so that a priori modeling of the background covariances becomes much more difficult.

To facilitate the comparison of the two approaches, we will consider here only simulated radar observations, and all our experiments will assume a perfect forecast model. Even with these simplifications and using a limited computational domain, the problem is still large enough that computational constraints are acute and both schemes involve compromises relative to theoretically optimal approaches. Thus, our comparison is between specific implementations of each scheme and intermingles the effects of such practical compromises in the schemes and fundamental differences in how the two algorithms perform in convective-scale assimilation. Nevertheless, this comparison is still of interest both as guidance for the development of future convective-scale assimilation systems, and as the first comparison of 4DVAR and the EnKF for a realistic flow.

The results for both 4DVAR and the EnKF could also potentially be improved through further tuning of each algorithm. Since the 4DVAR scheme used here has enjoyed a longer period of development than that of the EnKF, it is possible that further tuning of the EnKF could change the comparison with 4DVAR significantly. Our experience, however, suggests that benefits of further tuning will not be huge and will not change our conclusions.

The EnKF presented here clearly provides probabilistic information about both the analysis and forecasts, whereas the 4DVAR scheme, as implemented, does not. This is arguably a practical advantage of the EnKF. In principle, however, 4DVAR can be extended to be the basis for an ensemble forecasting system, for example, through the use of the Hessian of the cost function in initializing the ensemble perturbations. We have tried to confine the comparison of the schemes to those aspects that are available for both implementations.

The organization of the paper is as follows: first the methodology for the comparison of the two methods is detailed in section 2. In section 3, the 4DVAR and the EnKF algorithms are described, with emphasis on the details of each implementation. In addition, new features with respect to previous works (Snyder and Zhang 2003; Zhang et al. 2004) are introduced, namely, the assimilation of the rainwater content and the use of a correlation function to localize the ensemble covariances. The assimilation results are presented and compared in section 4. The effect of assimilating the rainwater content is examined. The performance of the two methods is also compared when the assimilations begin both at the cumulus stage and at the mature stage of a simulated storm. The effect of observational time dif- 
ferences in a radar volume owing to sequential scanning was studied by Sun and Crook (1998). This problem is revisited in the context of the EnKF and contrasted with the results of 4DVAR. We discuss the results and summarize the findings in section 5 .

\section{Methodology}

The cloud model of Sun and Crook (1997) is used in this study to generate a control simulation of a supercell storm and as the assimilation model for 4DVAR and the EnKF. Thus, the two methods are compared here in a perfect-model context. The prognostic variables are the velocity components $(u, v, w)$, the liquid water potential temperature $\left(\theta_{l}\right)$, the rainwater mixing ratio $\left(q_{r}\right)$, and the total water mixing ratio $\left(q_{t}\right)$, which is the sum of $q_{r}$ and the vapor and cloud water contents. The model has 2-km horizontal resolution and $500 \mathrm{~m}$ between vertical levels. The domain of $140 \times 140 \times 17.5 \mathrm{~km}^{3}$ is large enough to mitigate the effect of the boundaries on the simulated storm within the time the assimilations are performed. The model equations are integrated with a time step of $5 \mathrm{~s}$.

The initialization of the control simulation begins with the 0000 UTC 25 May 1997 Oklahoma City, Oklahoma, sounding. This sounding is particularly favorable to the development of supercell storms and is depicted in Snyder and Zhang (2003). The original wind sounding is used here, whereas $7 \mathrm{~m} \mathrm{~s}^{-1}$ was subtracted from the $u$ component at all levels in Snyder and Zhang (2003) and Zhang et al. (2004) to keep the storm within their smaller domain. A warm bubble is then superimposed on the horizontally uniform fields determined by this environmental sounding. The warm bubble is centered at $1.25-\mathrm{km}$ altitude, is $16 \mathrm{~km}$ wide and $2 \mathrm{~km}$ deep, and is $1^{\circ} \mathrm{C}$ warmer than the environment. There is neither precipitation nor cloud in the initial conditions, and the humidity field is horizontally homogeneous as prescribed by the sounding.

Observations of radial velocity $V_{r}$ and rainwater mixing ratio $q_{r}$ are simulated every $5 \mathrm{~min}$ (the approximate temporal resolution of the U.S. operational radar network) from the control simulation at those grid points where $q_{r}$ exceeds $0.13 \mathrm{~g} \mathrm{~kg}^{-1}$, or approximately $12 \mathrm{dBZ}$, as seen by a single radar located at the southwest corner of the domain. The fall speed of the precipitation is taken into account in the calculation of the radial velocities. Thus, the radial velocity is a weakly nonlinear function of the rainwater content $q_{r}$ in addition to the three wind components. A $1 \mathrm{~m} \mathrm{~s}^{-1}$ random Gaussian noise is added to the radial velocities before the assimilation, and this value is also used as the observationerror standard deviation for the radial-velocity obser- vations in both assimilation algorithms. No noise is added to the $q_{r}$ observations, although a standard deviation of $0.2 \mathrm{~g} \mathrm{~kg}^{-1}$ is assumed for the $q_{r}$ observation errors in both 4DVAR and the EnKF. This corresponds to having relatively little confidence in small rainwater contents and great confidence in large rainwater contents and was chosen to obtain the best results when assimilating $q_{r}$. Both 4DVAR and the EnKF analyses are almost insensitive to the small noise in the radial-velocity observations.

\section{Assimilation algorithms}

This section discusses the specifics of the two assimilation schemes. More general background on the algorithms can be found in Sun and Crook (1997) and Snyder and Zhang (2003).

\section{a. The 4DVAR algorithm}

The 4DVAR analysis $\mathbf{x}^{\mathrm{a}}$ is obtained through the minimization of a cost function $J$ that measures the misfit between the model trajectory $H_{i}(\mathbf{x})$ and the observations $\mathbf{y}_{i}$ at a series of times $t_{i}, i=1, \ldots, n$. The model error is neglected and the model is thus used as a strong constraint. The cost function is given by

$$
\begin{aligned}
J(\mathbf{x})= & \left(\mathbf{x}-\mathbf{x}^{\mathrm{b}}\right)^{\mathrm{T}} \mathbf{B}^{-1}\left(\mathbf{x}-\mathbf{x}^{\mathrm{b}}\right) \\
& +\sum_{i}\left[H_{i}(\mathbf{x})-\mathbf{y}_{i}\right]^{\mathrm{T}} \mathbf{R}^{-1}\left[H_{i}(\mathbf{x})-\mathbf{y}_{i}\right]+J_{p},
\end{aligned}
$$

where $\mathbf{x}$ is the model state at $t_{0}$ (the beginning of the assimilation window), $\mathbf{x}^{\mathrm{b}}$ is a background or prior estimate of $\mathbf{x}$, and has error covariance matrix $\mathbf{B}, \mathbf{R}$ is the observational-error covariance matrix, and $H_{i}$ is the generalized observational operator that includes the full nonlinear model integration from $t_{0}$ to $t_{i}$ and the full nonlinear operator to convert from model space to observational space. The term $J_{p}$ consists of a penalty on 3D divergence and of spatial and temporal smoothing constraints, which are described in Sun and Crook (1994) and in Lin et al. (2002).

The minimization of the cost function is achieved by adjusting the model initial condition $\mathbf{x}$ at $t_{0}$. The minimization proceeds by integrating the full nonlinear model through the end of the assimilation window to evaluate the cost function. Then the adjoint model is integrated backward in time to give the variation of the cost function with respect to $\mathbf{x}$. This information is used iteratively in a descent algorithm, which is terminated after 100 iterations in all experiments here. (Thus, the minimization may stop before it is completely converged.) The solution at the end of the assimilation 
window becomes the first guess for the minimization in the next assimilation cycle.

The forecast-error covariance matrix $\mathbf{B}$ is difficult to model, especially at the convective scale where balance constraints cannot be applied. For this reason, this 4DVAR scheme relies mainly on observations, and the role of the background-error covariance matrix is to control the analysis in data voids. This is accomplished as follows. First, $\mathbf{x}^{\mathrm{b}}$ is simply the environmental sounding. Thus, the analysis from the previous cycle is only used as a first guess for the minimization. Second, the covariances between model variables are neglected in B, which becomes a diagonal matrix. It should be noted that spatial correlations for $\mathbf{x}^{\mathrm{b}}$ are still implicit in $J$, however, because of the spatial smoothness constraints in $J_{p}$. Sun and Crook (2001) found that the effect of the spatial smoothness constraint is equivalent to a homogeneous correlation function implemented through the background-error covariance matrix $\mathbf{B}$. Third, the background-error variances are set to infinity wherever observations are available, thereby giving no weight to the background in those regions, while they remain finite in regions where no radar observations exist.

Thus, the covariances between model variables do not appear explicitly in $J$. They are achieved implicitly through the numerical model (as represented in $H_{i}$ ). The background term controls the analysis in data-void areas.

\section{b. An ensemble Kalman filter}

Unlike 4DVAR, the EnKF assimilates the observations sequentially. Given observations $\mathbf{y}_{i}$ at time $t_{i}$, the scheme requires an ensemble of $n_{e}$ forecasts from the previous analysis time, $t_{i-1}$. The EnKF updates $\overline{\mathbf{x}}_{i}^{\mathrm{f}}$, the ensemble mean of those forecasts, according to

$$
\overline{\mathbf{x}}_{i}^{\mathrm{a}}=\overline{\mathbf{x}}_{i}^{\mathrm{f}}+\tilde{\mathbf{K}}\left[\mathbf{y}_{i}-H\left(\overline{\mathbf{x}}_{i}^{\mathrm{f}}\right)\right],
$$

where $\overline{\mathbf{x}}_{i}^{\mathrm{a}}$ is the ensemble-mean analysis at $t_{i}$, and $H$ is as in 4DVAR except it does not include propagation of the state in time.

The matrix $\tilde{\mathbf{K}}$ is a generalization of the Kalman gain matrix

$$
\mathbf{K}=\mathbf{P}_{i}^{\mathrm{f}} \mathbf{H}^{\mathrm{T}}\left(\mathbf{H} \mathbf{P}_{i}^{\mathrm{f}} \mathbf{H}^{\mathrm{T}}+\mathbf{R}\right)^{-1},
$$

where $\mathbf{P}_{i}^{\mathrm{f}}$ is the forecast-error covariance matrix at $t_{i}$, and $\mathbf{H}$ is the observational operator, which must be linear. To obtain $\tilde{\mathbf{K}}$, the covariance matrices in the definition (3) of $\mathbf{K}$ are replaced by sample covariances based on the ensemble; that is, $\mathbf{P}_{i}^{\mathbf{f}} \mathbf{H}^{\mathrm{T}} \rightarrow\left(n_{e}-1\right)^{-1} \sum_{j}\left(\mathbf{x}_{i, j}^{\mathbf{f}}\right.$ $\left.-\overline{\mathbf{x}}_{i}^{\mathrm{f}}\right)\left[H\left(\mathbf{x}_{i, j}^{\mathrm{f}}\right)-\overline{H\left(\mathbf{x}_{i}^{\mathrm{f}}\right)}\right]^{\mathrm{T}}$ and $\mathbf{H P}_{i}^{\mathrm{f}} \mathbf{H}^{\mathrm{T}} \rightarrow\left(n_{e}-1\right)^{-1}$ $\Sigma_{j}\left[H\left(\mathbf{x}_{i, j}^{\mathrm{f}}\right)-\overline{H\left(\mathbf{x}_{i}^{\mathrm{f}}\right)}\right]\left[H\left(\mathbf{x}_{i, j}^{\mathrm{f}}\right)-\overline{H\left(\mathbf{x}_{i}^{\mathrm{f}}\right)}\right]^{\mathrm{T}}$, where overbars denote ensemble averages. Note that in these expres- sions, the full nonlinear observational operator $H$ is used.

The EnKF also updates the deviations of the forecast ensemble from $\overline{\mathbf{x}}_{i}^{\mathrm{f}}$ to account for the reduction of uncertainty given the observations $\mathbf{y}_{i}$. This procedure produces an analysis ensemble at $t_{i}$, which then serves as initial conditions for the ensemble forecast to $t_{i+1}$. The update of the ensemble members used here follows the "square root" method of Whitaker and Hamill (2002).

For linear systems (model and observational operators) with Gaussian errors, the EnKF converges to the Kalman filter as the ensemble size becomes large. In that case, the EnKF analysis $\overline{\mathbf{x}}_{i}^{\text {a }}$ given by (2) minimizes the cost function

$$
\begin{aligned}
J(\mathbf{x})= & \left(\mathbf{x}-\overline{\mathbf{x}}_{i}^{\mathrm{f}}\right)^{\mathrm{T}}\left(\mathbf{P}_{i}^{\mathrm{f}}\right)^{-1}\left(\mathbf{x}-\overline{\mathbf{x}}_{i}^{\mathrm{f}}\right) \\
& +\left[H(\mathbf{x})-\mathbf{y}_{i}\right]^{\mathbf{T}} \mathbf{R}^{-1}\left[H(\mathbf{x})-\mathbf{y}_{i}\right] .
\end{aligned}
$$

Comparing (4) with (1) shows that the 4DVAR cost function (as implemented here) includes an additional term, $J_{p}$, and uses $\mathbf{x}^{\mathrm{b}}$ and its assumed covariance matrix rather than a prior forecast. [Although the 4DVAR $J(\mathbf{x})$ fits observations over the entire assimilation window while (4) involves only observations at a single time, sequential assimilation with the Kalman filter in the linear, Gaussian case yields an identical analysis at the end of the window (Lorenc 1986).] Thus, in the experiments that follow, the 4DVAR and EnKF results will differ because of differences in the underlying cost functions, and because of the nonlinearities inherent in moist-convective dynamics and in the observational operators, in addition to sampling errors in the EnKF and incomplete minimization for 4DVAR.

Unlike the 4DVAR scheme used here, the forecasterror covariance matrix is meant to play a central role in the assimilation with the EnKF. The forward propagation of the ensemble between observation times incorporates the information on the dynamics into the ensemble and then $\mathbf{P}^{f}$ is derived from the forecast ensemble with a single further assumption, namely that the covariances are spatially local as described in section $3 \mathrm{~b}(2)$ below.

All the experiments with the EnKF use an ensemble size of $n_{e}=100$. This was chosen in part to make the number of forward model integrations identical to 4DVAR, which uses 100 iterations in the minimization of $J(\mathbf{x})$. The computational cost of the two schemes is thus also broadly comparable.

Because sampling errors and other effects tend to make the ensemble variance systematically too small in the EnKF, many implementations of the EnKF also employ techniques to increase the ensemble variance. No such techniques are used here. In particular, experi- 
ments revealed that the "covariance relaxation" technique of Zhang et al. (2004) degraded the quality of the EnKF analyses. We believe that covariance relaxation is less effective here than in the Zhang et al. results because of our use of localized and spatially correlated noise to generate the initial ensemble perturbations, which is described in the subsection below. In comparison to the spatially white perturbations used in Zhang et al., these perturbations maintain the ensemble variance better and produce more realistic structure in the early assimilation cycles.

\section{1) ENSEMBLE INITIAL CONDITIONS}

Before any observations are assimilated, the initial ensemble should reflect all prior information and its uncertainty. Beyond the use of the (known) environmental sounding for $u, v, \theta_{l}$, and $q_{t}$, however, the initial conditions for the ensemble are determined only qualitatively by other physical information, such as spatial smoothness or the requirement that rain be present in the model only in regions of significant reflectivity. In their basic experiments, Snyder and Zhang (2003) ignored such prior physical information and simply generated initial ensemble members by adding random noise, which was independent at each grid point and for each variable, to the environmental sounding.

Here, we construct ensemble initial conditions that are both spatially smooth and localized where reflectivity observations show rain is present. For each of the six model variables $\phi$, the fields for the ensemble members are perturbed from the ensemble mean $\bar{\phi}$ using the following relation:

$$
\begin{aligned}
\phi(l, m, n)= & \bar{\phi}(l, m, n)+\sum_{(i, j, k) \in S} r(i, j, k) \exp \left(-\frac{|i-l|}{l_{h}}\right. \\
& \left.-\frac{|j-m|}{l_{h}}-\frac{|k-n|}{l_{v}}\right)
\end{aligned}
$$

where indices $(l, m, n)$ include all model grid points of the computational domain. Each $r(i, j, k)$ is a random number sampled independently from a normal distribution with zero mean and variance $\sigma_{\phi}^{2}$, where $\sigma_{\phi}^{2}$ is chosen for each variable to obtain an appropriate initial ensemble spread. The set of gridpoint indices $S$ included in the sum in (5) is determined by the available reflectivity observations, as will be described below. The horizontal correlation length scale $l_{h}$ is $4 \mathrm{~km}$ and the vertical correlation length scale $l_{v}$ is $2 \mathrm{~km}$, both of which were specified according to the correlation length scales in the control simulation and by trial and error. The idea is that spatially smooth perturbations should be inserted in the initial ensemble only in the vicinity of observation locations.
The first set of observations is also used to initialize the ensemble mean. For the cases where $q_{r}$ is assimilated, $q_{r}$ is initialized with its observed value. Otherwise, the initial ensemble mean of $q_{r}$ is set to zero. The liquid water potential temperature $\theta_{l}$ initial ensemble mean is modified according to the definition

$$
\theta_{l}=\theta\left(1-\frac{L_{v}}{c_{p} T}\left(q_{c}+q_{r}\right)\right),
$$

assuming zero cloud content $q_{c}$. The contribution of $q_{r}$ to $\theta_{l}$ can be as large as $-20 \mathrm{~K}$, and should thus be taken into account in the initial conditions of $\theta_{l}$. Then, the model variables $u, v, \theta_{l}, q_{r}$, and $q_{t}$ are perturbed as in (5), with the summation running over the set $S$ of all gridpoint indices $(i, j, k)$ where an observation is available. All the negative values produced for $q_{r}$ during this process are set to zero. The liquid water potential temperature $\theta_{l}$ and $q_{t}$ are adjusted according to the added perturbation on $q_{r}$. Finally, the vertical velocity field is obtained by integrating the anelastic continuity equation with the perturbed horizontal velocity fields.

\section{2) LocALiZATION}

The limited ensemble size results in sampling error and means that small correlations between widely separated state variables are typically poorly estimated (Houtekamer and Mitchell 1998; Hamill et al. 2001). For this reason, each observation is allowed to influence only state variables located within a certain distance of the observation. This localization also reduces the computational cost of the analysis in the EnKF because only a portion of the model state is updated.

More precisely, the localization is applied as in Houtekamer and Mitchell (2001) by taking the Schur (or element by element) product of a compactly supported correlation function, which depends only on the $3 \mathrm{D}$ distance between a given observation and the state variables, with the sample covariances from the ensemble. The correlation function is the compactly supported fifth-order piecewise rational function of Gaspari and Cohn (1999). Defining a correlation length scale $l_{c}$ as in Daley (1991, p. 110),

$$
l_{c} \equiv \frac{1}{\left[-f^{\prime \prime}(0)\right]^{1 / 2}} ;
$$

this function $f$ is approximately equal to 0.6 at one correlation length scale $l_{c}$ and is zero for distances greater than $2 \sqrt{10 / 3} l_{c}$ (see section $4 c$ of Gaspari and Cohn 1999).

We have explored the optimal correlation length scale for the localization in the assimilation from 20 to 60 min where both $V_{r}$ and $q_{r}$ are assimilated. Figure 1 


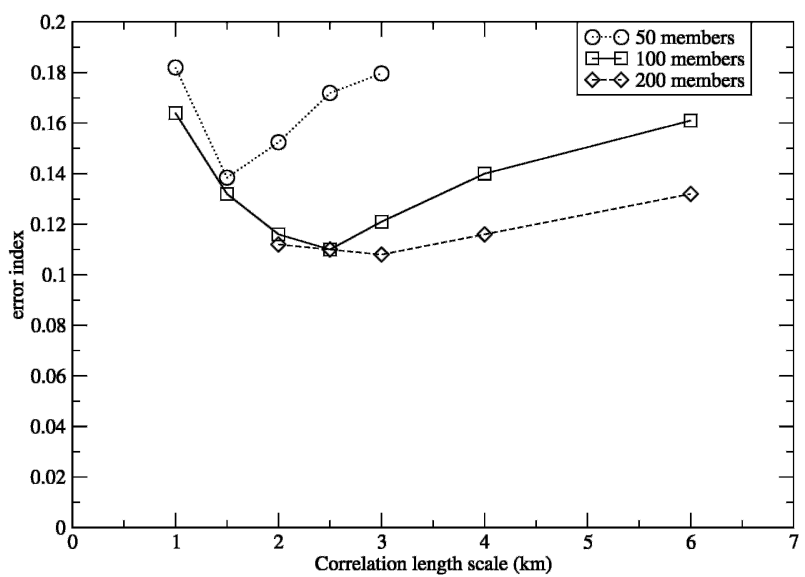

FIG. 1. Error index of the EnKF analysis as a function of the correlation length scale for ensembles with 50, 100, and 200 members. The error index is calculated for assimilations from 20 to 60 min in which both $V_{r}$ and $q_{r}$ are assimilated. See Eq. (8) in the text for an explicit formula of the error index.

shows an analysis error index, which is calculated as follows. Defining the root-mean-square error (over the entire domain) for model variable $i$ (in $\mathrm{m} \mathrm{s}^{-1}$ for the wind components, in $\mathrm{K}$ for $\theta_{l}$, and in $\mathrm{g} \mathrm{kg}^{-1}$ for $q_{r}$ and $q_{t}$ ) at time $j$ as $\xi_{i, j}$, the error index $\varepsilon$ is

$$
\varepsilon=\sqrt{\sum_{j=t_{o}}^{t_{f}} \sum_{i=1}^{6}\left(\xi_{i, j}\right)^{2}},
$$

where the subscript $i$ runs over the six model variables and subscript $j$ over analysis times. This error index is computed as a function of $l_{c}$ for ensembles having 50, 100, and 200 members. The results in Fig. 1 are based on a single realization of the initial ensemble for each ensemble size. As shown by Snyder and Zhang (2003) (and as will be seen later in Table 2, section 4), other realizations of the initial ensemble will produce some variation in these rms errors.

Overall, Fig. 1 is broadly similar to Fig. 4 of Houtekamer and Mitchell (2001): Increasing the ensemble size from 50 to 100 members improves the results significantly, while a further doubling of the ensemble size to 200 members yields only marginal improvements. This justifies our choice of a 100-member ensemble. In addition, the error index is minimum and its variation is only $5 \%$ when $l_{c}$ is between 2 and $3 \mathrm{~km}$ for an ensemble of 100 members. A correlation length scale of $2 \mathrm{~km}$ is chosen to make the EnKF as efficient as possible, given the ensemble size of $n_{e}=100$. For $l_{c}=2 \mathrm{~km}$, the influence of an observation goes to zero at a distance of $7.3 \mathrm{~km}$.

Since previous studies with the EnKF (Snyder and Zhang 2003; Zhang et al. 2004) have localized by simply setting the influence of an observation to zero beyond a given cutoff radius, we also measured the relative benefits of using the aforementioned correlation function. For the same 50-member ensemble used for the results shown in Fig. 1 and with the cutoff radius approach, the lowest error index we obtained is 0.22 with a $4-\mathrm{km}$ cutoff radius of influence. Thus, the use of the correlation function greatly improves the results relative to the experiments where the cutoff radius is employed.

\section{Comparison between the EnKF and MDVAR}

The performance of the EnKF and 4DVAR algorithms will now be compared. The 4DVAR analysis uses a 10-min assimilation window. The initial ensemble mean in the EnKF is also used as the first guess for the 4DVAR minimization in the first cycle. To help interpret the results, Table 1 shows the number of available observations as a function of time during the assimilation.

\section{a. Effect of assimilating the rainwater content}

For these experiments, the assimilations begin at $t=$ 20 min into the control simulation, at which time precipitation has just begun to form, and end after $40 \mathrm{~min}$, at $t=60 \mathrm{~min}$. The first assimilation window of 4DVAR is between 20 and 30 min into the simulation and includes observations at 20, 25, and $30 \mathrm{~min}$. The EnKF is initialized at $20 \mathrm{~min}$, and the first data are assimilated at 20 min by the EnKF.

In what follows, we will compare the 4DVAR and EnKF analyses only at the end of the 4DVAR assimilation windows. This is because at times before the end of its assimilation window, the 4DVAR results depend

TABLE 1. Number of observational grid points as a function of time ( $\min )$. At each grid point, radial velocity and rainwater content are available.

\begin{tabular}{cc}
\hline Time $(\min )$ & Observations \\
\hline 20 & 17 \\
25 & 150 \\
30 & 613 \\
35 & 1114 \\
40 & 1490 \\
45 & 1800 \\
50 & 2063 \\
55 & 2195 \\
60 & 2316 \\
65 & 2521 \\
70 & 2677 \\
75 & 2945 \\
80 & 3186 \\
85 & 3412 \\
90 & 3492 \\
\hline
\end{tabular}


on the assimilation of future observations while the EnKF analysis does not. In the figures, 4DVAR analysis errors will be shown only at the end of each 10-min assimilation window. For the EnKF, analysis errors will be shown at each observation time, together with errors for the 5-min forecast from the previous analysis in order to indicate the reduction (or increase) of error by the assimilation of observations with the EnKF.

Figure 2 shows the rms error (over the entire domain) of the three wind components when only the radial velocities are assimilated with the EnKF and 4DVAR. The 4DVAR assimilation produces a better analysis of the horizontal wind components over this period. The analysis of the vertical motion is also better with 4DVAR except at 50 and $60 \mathrm{~min}$ where the EnKF gives better results.

Figure 3 is similar to Fig. 2 except that the rainwater content $q_{r}$ is also assimilated. The results with the EnKF are much better than in the previous experiment (except at 30 min where the assimilation of $q_{r}$ results in larger errors for the wind components), while those with 4DVAR are only slightly improved. With the assimilation of both $V_{r}$ and $q_{r}$, all wind-component errors at 50 and $60 \mathrm{~min}$ are smaller with the EnKF than they are with 4DVAR, while 4DVAR still improves on the EnKF for the horizontal wind components at 30 and 40 min. For this experiment, the vertical motion from the EnKF analysis has a smaller rms error than that from 4DVAR starting as soon as $40 \mathrm{~min}$.

Figure 4 shows the error in the thermodynamic and condensate variables when only the radial velocities are assimilated. The 4DVAR scheme again has a small advantage over the EnKF at $30 \mathrm{~min}$, except for $q_{r}$. For the

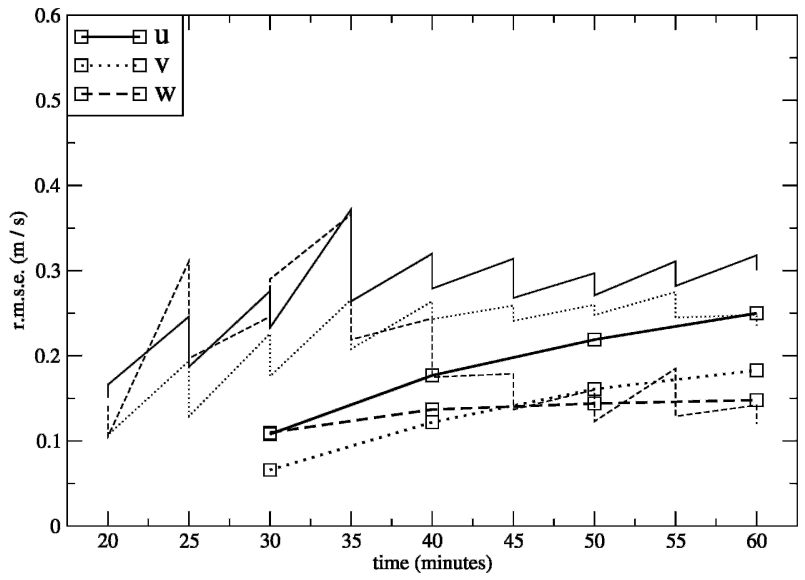

FIG. 2. The forecast and analysis rmse of the three wind components during the assimilation with the EnKF (thin lines). The 4DVAR wind analysis root-mean-square error at the end of each assimilation window is given by the thick lines with the squares. Only the radial velocities are assimilated.

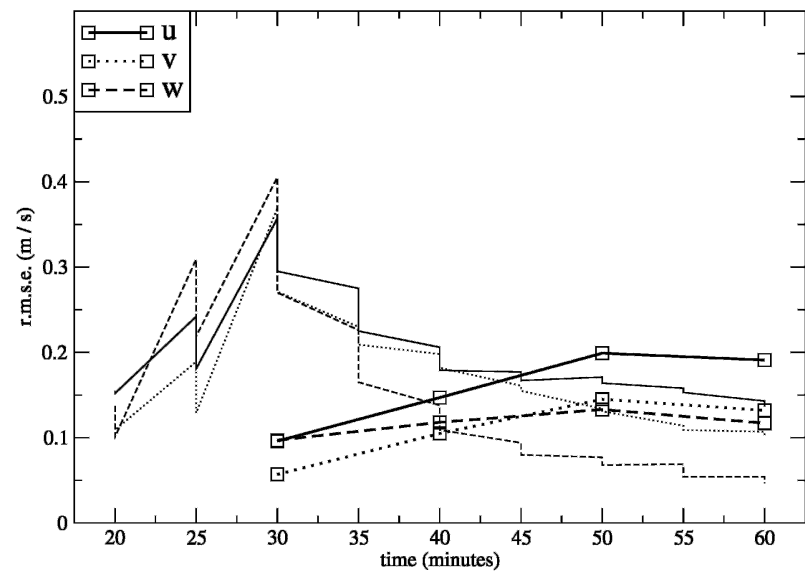

FIG. 3. As in Fig. 2, but both the radial velocity, $V_{r}$, and the rainwater content, $q_{r}$, are assimilated.

analyses at 40, 50, and $60 \mathrm{~min}$, the EnKF and 4DVAR give similar results for the condensates, and the $\theta_{l}$ analysis is better with the EnKF.

Figure 5 is the same plot as Fig. 4 except that the rainwater content $q_{r}$ is also assimilated. The errors are reduced in both methods relative to the case with only observations of the radial velocity, but the reduction is significantly larger for the EnKF, so that the 4DVAR analyses at 60 min have roughly 3 times the rms error of the EnKF analyses when both $V_{r}$ and $q_{r}$ are assimilated. The assimilation of $q_{r}$ has a negative impact on the temperature up to $30 \mathrm{~min}$ for the EnKF analyses. Nevertheless, the EnKF analysis error of $\theta_{l}$ is already smaller than the 4DVAR analysis error by $40 \mathrm{~min}$.

The performance of the EnKF can vary from one

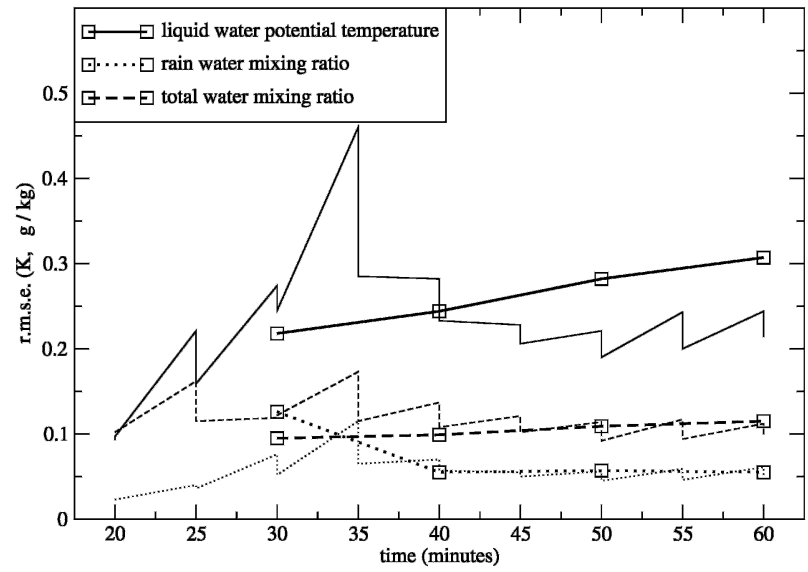

FIG. 4. The forecast and analysis rmse of the liquid water potential temperature $\theta_{l}$, the rainwater content $q_{r}$, and the total water content $q_{t}$ of the EnKF (thin lines) and 4DVAR (thick lines) analyses. Only the radial velocities are assimilated. 


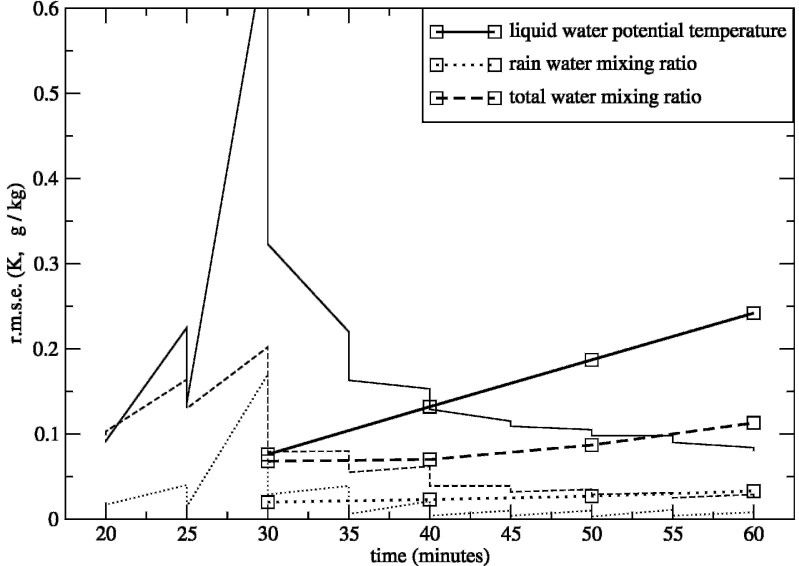

FIG. 5. As in Fig. 4, but both the radial velocity, $V_{r}$, and the rainwater content, $q_{r}$, are assimilated.

randomly chosen realization of the initial ensemble to the next [see Snyder and Zhang (2003), their Fig. 5], but the assimilation of the rainwater content $q_{r}$ reduces that variability. Using a 25 -member ensemble and assimilating only radial velocities, the analysis error index is relatively high in a few realizations (two of seven; Table 2 ) and the performance of the filter is very variable. This is due, in part, to the small ensemble size $(25 \mathrm{mem}-$ bers) used here. When $q_{r}$ is assimilated in the same experiments, the error index is always smaller than for the same experiment without the assimilation of $q_{r}$. This is especially true for the two experiments where the error index is close to 1.3 without the assimilation of $q_{r}$. The results are also much less variable when $q_{r}$ is assimilated. The variability of the results is greatly reduced for an ensemble size of 100 members.

\section{b. Assimilation beginning at the mature stage}

We turn now to the case where the assimilation begins at the mature stage of the storm. Thus, at the be-

TABLE 2. Error index [Eq. (8)] of the EnKF analysis for ensembles with 25 and 100 members after different realizations. For each initial ensemble (realization), the assimilation is performed without and with $q_{r}$. The statistics are calculated on a $34 \times 34$ gridpoint horizontal domain.

\begin{tabular}{cccccc}
\hline \hline & \multicolumn{2}{c}{25 members } & & \multicolumn{2}{c}{100 members } \\
\cline { 2 - 3 } \cline { 5 - 6 } Expt No. & $V_{r}$ only & $V_{r}$ and $q_{r}$ & & $V_{r}$ only & $V_{r}$ and $q_{r}$ \\
\hline 1 & 1.30 & 0.29 & & 0.24 & 0.17 \\
2 & 0.40 & 0.33 & & 0.19 & 0.18 \\
3 & 0.29 & 0.27 & & 0.23 & 0.19 \\
4 & 0.64 & 0.30 & & 0.20 & 0.17 \\
5 & 0.35 & 0.28 & & 0.22 & 0.18 \\
6 & 1.27 & 0.55 & & 0.19 & 0.18 \\
7 & 0.39 & 0.30 & & 0.21 & 0.17 \\
\hline
\end{tabular}

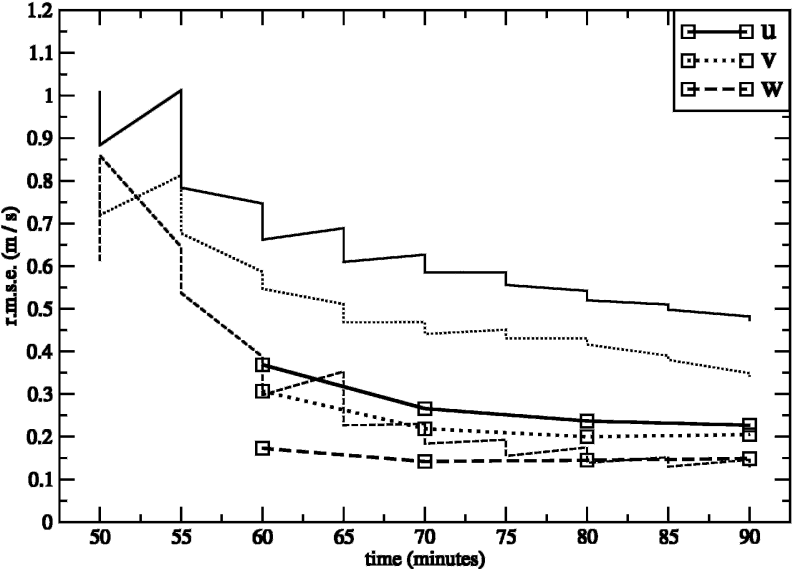

FIG. 6. The forecast and analysis rmse of the three wind components of the EnKF (thin lines) and 4DVAR (thick lines with squares) analyses. The radial velocity $V_{r}$ and the rainwater content $q_{r}$ are assimilated. The assimilation is started at the mature stage of the storm.

ginning of the assimilation, the ensemble mean for the EnKF and the first guess for the 4DVAR minimization are farther away from the control simulation than in the previous experiments. Both radial velocity and rainwater content are assimilated with the two methods. The EnKF is initialized at $50 \mathrm{~min}$ while the first assimilation window of 4DVAR is between 50 and $60 \mathrm{~min}$, both assimilations including observations at $50 \mathrm{~min}$.

Except for the vertical velocity at 80 and $90 \mathrm{~min}$, the 4DVAR wind analysis in this case is better than that of the EnKF, as illustrated by Fig. 6, which shows the rms errors for the wind components. Figure 7 shows the rms analysis errors for the thermodynamic and condensate variables. For these variables, the errors in the EnKF analyses are smaller than those from 4DVAR, except for $\theta_{l}$ at $60 \mathrm{~min}$.

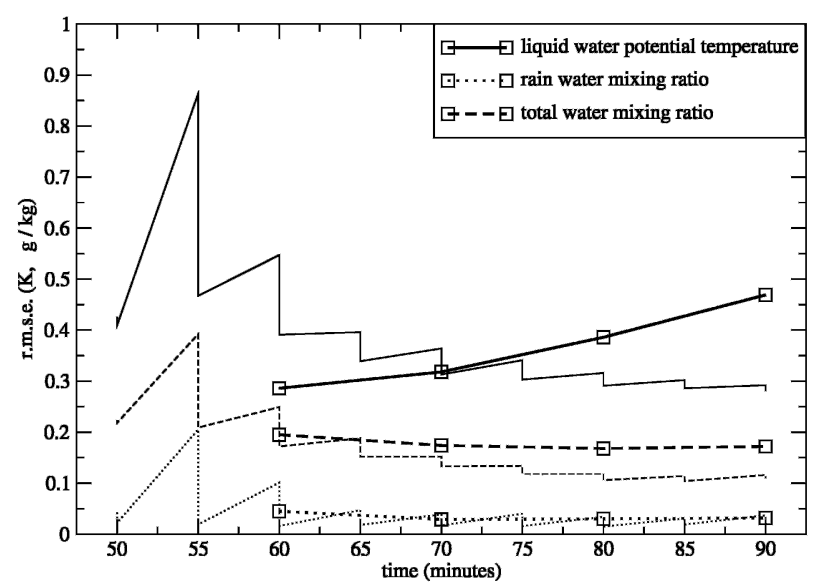

FIG. 7. As in Fig. 6 but for the liquid water potential temperature $\theta_{l}$, the rainwater content $q_{r}$, and the total water content $q_{t}$. 

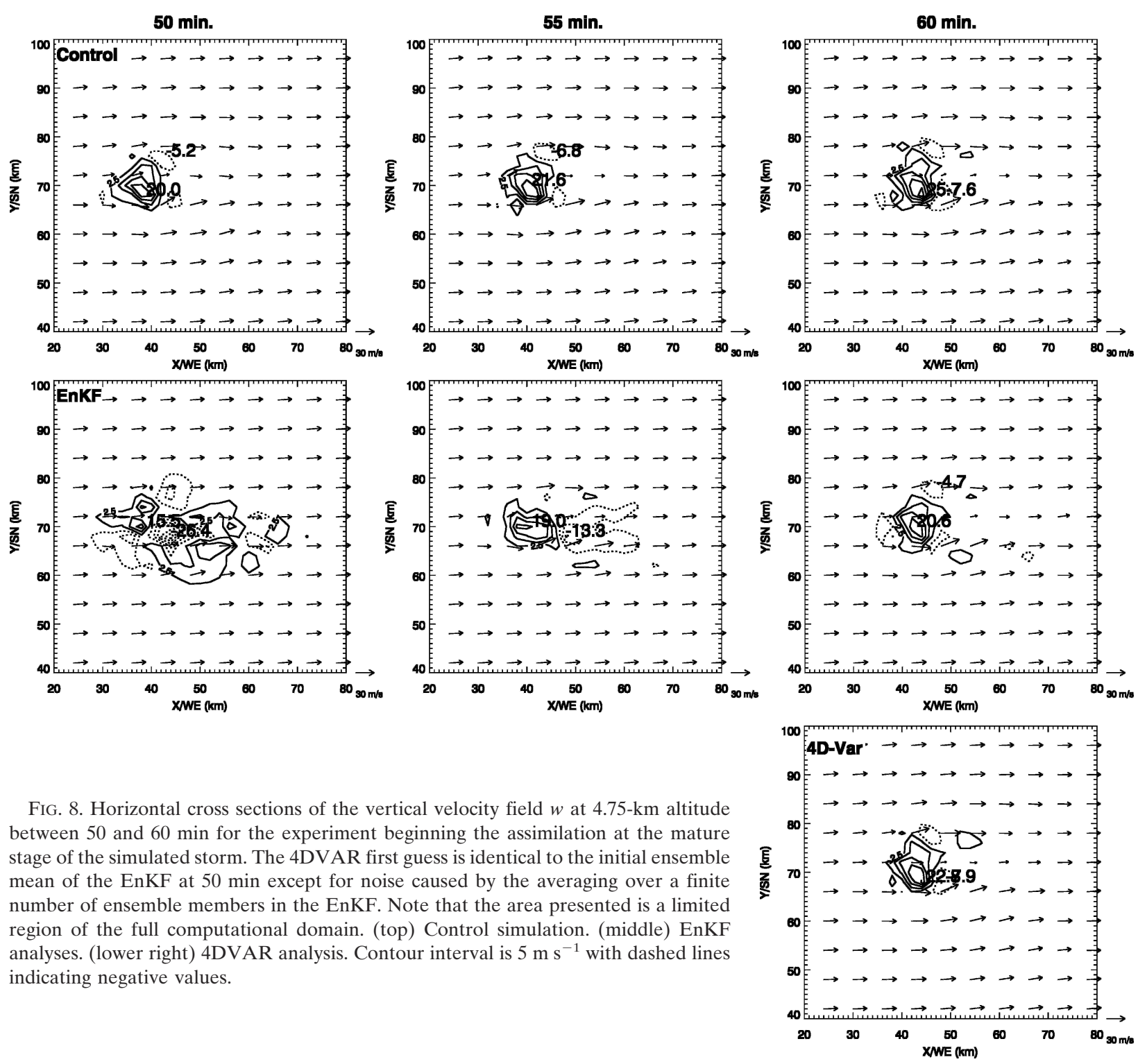

FIG. 8. Horizontal cross sections of the vertical velocity field $w$ at $4.75-\mathrm{km}$ altitude between 50 and $60 \mathrm{~min}$ for the experiment beginning the assimilation at the mature stage of the simulated storm. The 4DVAR first guess is identical to the initial ensemble mean of the EnKF at 50 min except for noise caused by the averaging over a finite number of ensemble members in the EnKF. Note that the area presented is a limited region of the full computational domain. (top) Control simulation. (middle) EnKF analyses. (lower right) 4DVAR analysis. Contour interval is $5 \mathrm{~m} \mathrm{~s}^{-1}$ with dashed lines indicating negative values.

Figure 8 shows the vertical velocity field $w$ at $4.75-\mathrm{km}$ altitude during the first assimilation window of 4DVAR. The 4DVAR first guess (at $50 \mathrm{~min}$ ) is zero vertical motion. In the EnKF, the vertical velocity is initialized by integration of the continuity equation. Since the initial (prior) ensemble-mean horizontal wind is uniform, the initial mean vertical motion is zero except for small variations arising from sampling errors. The assimilation in the EnKF at 50 min produces large analysis increments in $w$ because correlations between the vertical motion and the horizontal wind field (and thus the radial velocities) were created by the vertical integration of the continuity equation in the generation of the initial ensemble. The EnKF analysis at $50 \mathrm{~min}$ is still far from the control simulation and the root-mean- square analysis error is actually larger for the posterior than the prior EnKF estimate, as indicated in Fig. 6. At 55 min the EnKF assimilation becomes closer to the control simulation. After three sets of data have been assimilated (i.e., at $60 \mathrm{~min}$ ), both analyses contain a strong cell in the correct location. The maximum vertical velocity in the center of the storm, however, is still smaller in the EnKF analysis $\left(20.6 \mathrm{~m} \mathrm{~s}^{-1}\right)$ than in the control simulation $\left(25.7 \mathrm{~m} \mathrm{~s}^{-1}\right)$, while the 4DVAR analysis lies in between $\left(22.8 \mathrm{~m} \mathrm{~s}^{-1}\right)$.

The discrepancies between root-mean-square analysis errors in 4DVAR and in the EnKF at $60 \mathrm{~min}$ are interesting because this is the only time when the two algorithms use the same information. As discussed in section 3 , the two algorithms are not based on the same 
cost function, and for this reason, results from the two methods are expected to differ. In particular, the 4DVAR background-error variances are infinitely large in data regions and this feature cannot be represented within the EnKF. For this reason, the 4DVAR algorithm used in this study is not very sensitive to the error in the first guess for the minimization. (Since the minimization proceeds for a fixed number of iterations, there is some dependence on the first guess, which can be seen in comparing the results in Figs. 3 and 6 for the window between 50 and $60 \mathrm{~min}$.) On the contrary, the initial ensemble affects the performance of the EnKF. In what follows, we examine the extent to which the ensemble variance in regions of observations is an issue for the EnKF.

An indication that the EnKF is functioning properly is that the ensemble standard deviation should be close to the actual ensemble-mean error. One way of verifying this property is by studying the innovation (observation-minus-forecast) statistics. Defining the innovation vector at the $i$ th time by $\mathbf{d}_{i}=\mathbf{y}_{i}-H\left(\overline{\mathbf{x}}_{i}^{\mathrm{f}}\right)$, the expectation of the innovation matrix can be estimated from the ensemble as in Mitchell and Houtekamer (2000, section $3 a)$ :

$$
\begin{aligned}
\mathrm{E}\left[\mathbf{d}_{i} \mathbf{d}_{i}^{\mathrm{T}}\right] \approx & \left(n_{e}-1\right)^{-1} \sum_{j}\left[H\left(\mathbf{x}_{i, j}^{\mathrm{f}}\right)-\overline{H\left(\mathbf{x}_{i}^{\mathrm{f}}\right)}\right] \\
& \times\left[H\left(\mathbf{x}_{i, j}^{\mathrm{f}}\right)-\overline{H\left(\mathbf{x}_{i}^{\mathrm{f}}\right)}\right]^{\mathrm{T}}+\mathbf{R},
\end{aligned}
$$

where the index $j$ refers to an ensemble member.

Figure 9 shows the root mean of the trace of the innovation matrix [from the left-hand side of (9)] and the root mean of the ensemble variance plus the observational error variance [from the right-hand side of (9)] for (a) the radial velocities and (b) the rainwater content for the experiment beginning at the mature stage of the storm (the experiment shown in Figs. 6 and 7). Figure 9 also shows the same statistics after the analysis (lower points on the curves). Initially, we set the ensemble standard deviation to be larger than the innovation to mimic the fact that 4 DVAR has infinite background-error variance at the observation locations. This produces a close fit to the observations (at the level of the observational error) after the analysis at 50 min. After only a few assimilation cycles the two curves almost coincide, in agreement with (9). Meanwhile, the analysis residuals become very close to the observational error. The error of the mean also agrees with the ensemble standard deviation for other model variables (not shown). Thus, the ensemble spread from the EnKF yields a good estimate of the magnitude of the error of the ensemble mean through most of the assimilation, though it is somewhat too large in the first few cycles.

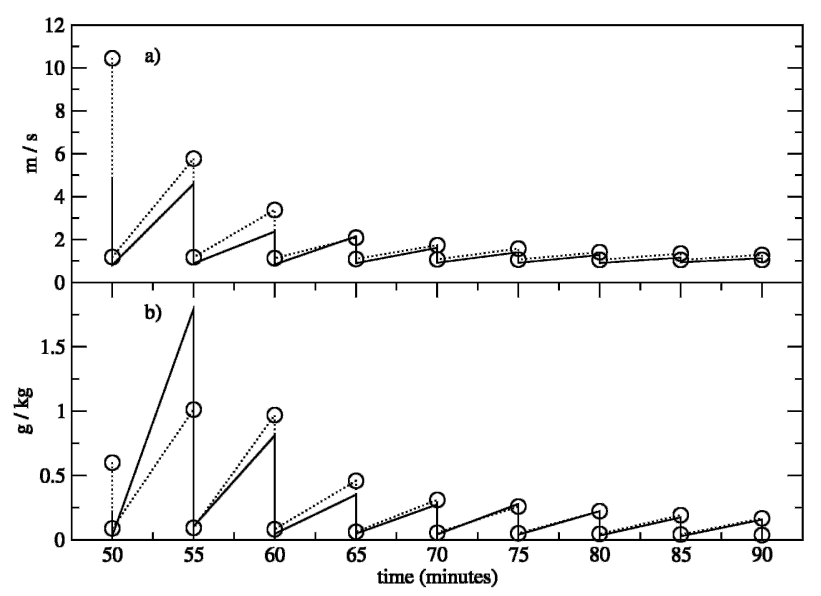

FIG. 9. Rms observation-minus-forecast and observation-minusanalysis residuals (full lines) for (a) radial velocity and (b) rainwater content in the EnKF experiment beginning at the mature stage of the storm. The dotted line with circles is the root mean of the ensemble variance plus the observational error variance [right-hand side of Eq. (9) in the text].

Additional experiments using doubled and halved initial ensemble variances (not shown) did not improve the EnKF results. The results of these tests (and that shown in Fig. 9) indicate that the performance of the EnKF relative to 4DVAR is not controlled by deficiencies in the magnitude of the ensemble spread, nor is it explained by the close fit to the observations that follows from the infinite background-error variances at observation locations in 4DVAR. We have also shown (see Fig. 1) that increasing the ensemble size from 100 to 200 improves the EnKF results only slightly. The differences between the 4DVAR and EnKF analyses at $60 \mathrm{~min}$ are therefore most likely related either to other deficiencies in the initial ensemble used for the EnKF, or to other differences in the cost functions for the two schemes, such as the temporal smoothness constraint used in 4DVAR, or to nonlinearities in the forecast evolution.

\section{c. Observational error due to observational time}

A frequent assumption in radar data assimilation is that all observations in a given radar volume, which are typically collected over a span of 5 min, are valid at a single time. Here we examine how this assumption influences the comparison of 4DVAR with the EnKF through two experiments.

In the first experiment all observations on a given model level are extracted from the control simulation every 10 s (i.e., every other model time step); this process begins with the lowest model level, and continues with each successively higher level until 5 min (or 60 
time steps) have elapsed, at which point observations from all remaining six vertical levels are extracted. Then all these observations are assimilated using 4DVAR and the EnKF as if they were simultaneous observations every $5 \mathrm{~min}$. For example, the assimilation schemes assume that the data collected between 20 and $25 \mathrm{~min}$ are valid at $25 \mathrm{~min}$. The EnKF is initialized at 25 min and the first assimilation window of the 4DVAR is between 20 and $30 \mathrm{~min}$. No data are assimilated at 20 min in either algorithm. The assimilations are cycled up to $60 \mathrm{~min}$.

The rms errors for the wind components are presented in Fig. 10. The 4DVAR (EnKF) analysis wind errors are approximately double (triple) those for the case where the assumption of simultaneous observations is true (Fig. 3). For the thermodynamic and condensate variables, the same conclusion can be drawn by comparing Figs. 11 and 5. Thus, 4DVAR analyses appear somewhat less sensitive to this kind of observational error, at least for the wind components, than the EnKF. This result can be explained by the combination of two effects. When the difference between the correct and assumed time is neglected, the first effect is that radial velocities have effectively larger errors than the assumed $1 \mathrm{~m} \mathrm{~s}^{-1}$ observational error standard deviations. Both algorithms suffer from this effect. The second effect is that the uncertainty (the ensemble variance) is reduced too much in the EnKF, resulting in too little ensemble spread for the following analysis cycle. The 4DVAR algorithm does not suffer from this second effect because its background-error variance is fixed in time. For the thermodynamic and condensate

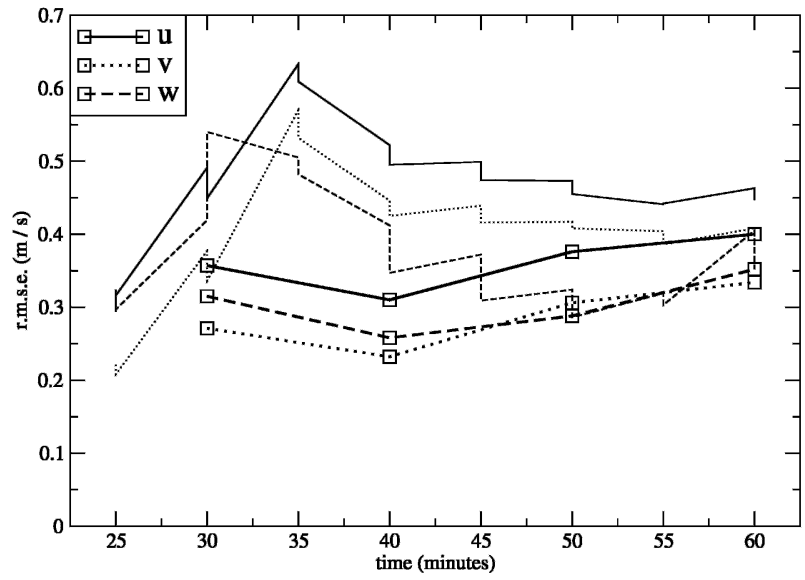

FIG. 10. The forecast and analysis rmse of the three wind components of the EnKF (thin lines) and 4DVAR (thick lines) analyses. The radial velocity $V_{r}$ and the rainwater content $q_{r}$ are assimilated. The data are collected at each $10 \mathrm{~s}$ and assimilated as if they were valid at each $5 \mathrm{~min}$.

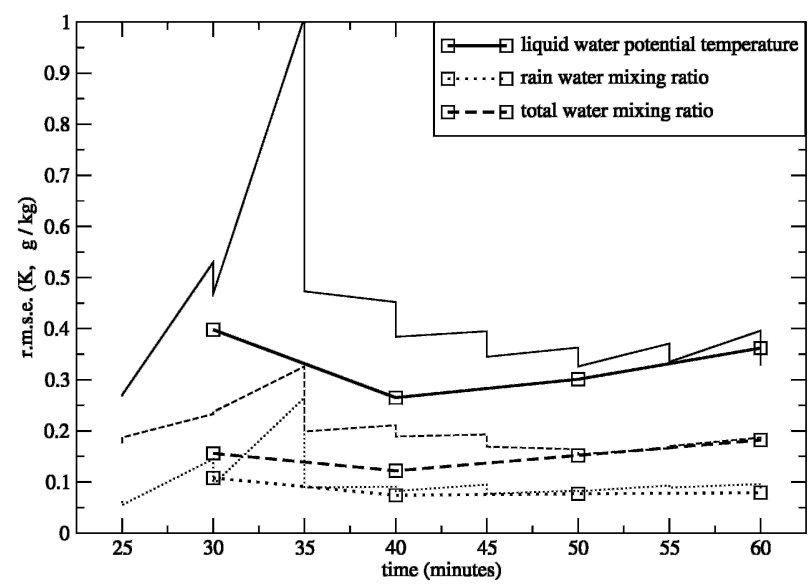

FIG. 11. As in Fig. 10 but for the liquid water potential temperature $\theta_{l}$, the rainwater content $q_{r}$, and the total water content $q_{t}$.

variables, however, the results with $4 \mathrm{DVAR}$ and the EnKF are very similar at 50 and $60 \mathrm{~min}$.

In the second experiment, the observations are assimilated at the correct time (i.e., at each $10 \mathrm{~s}$ or two time steps). The results are shown in Fig. 12 for the wind components and in Fig. 13 for the thermodynamic and condensate variables. For the EnKF, the assimilation of the data at the correct times paradoxically results in larger errors (cf. Figs. 12 and 10). This problem is particularly noticeable over the first 5-10 min, where the EnKF analysis of the wind field is often much worse than the prior 10 -s forecast. The 4DVAR assimilation, in contrast, does not suffer this effect.

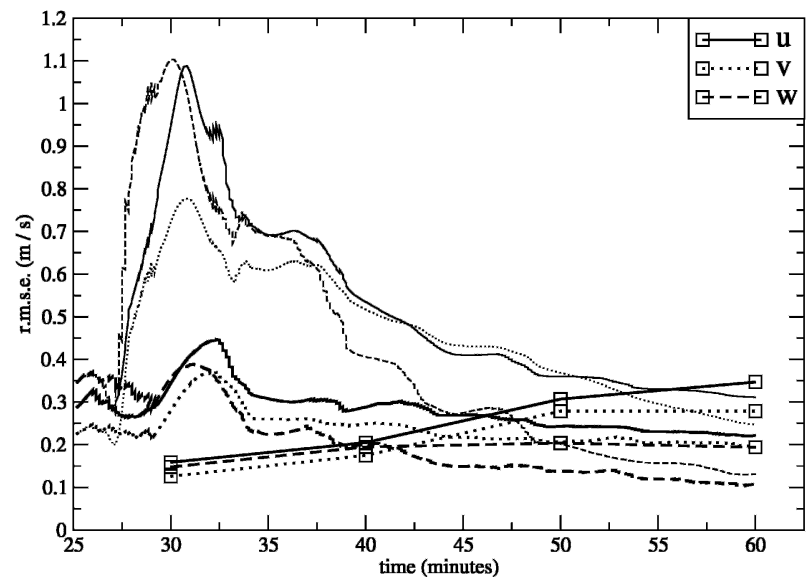

FIG. 12. The forecast and analysis rmse of the three wind components of the EnKF (thin lines) and 4DVAR (thick lines with squares) analyses. For these two experiments, the radial velocity $V_{r}$ and the rainwater content $q_{r}$ are assimilated. The data are assimilated at each $10 \mathrm{~s}$. The thick curves without the squares are for the EnKF experiment where only radial velocities are assimilated. 


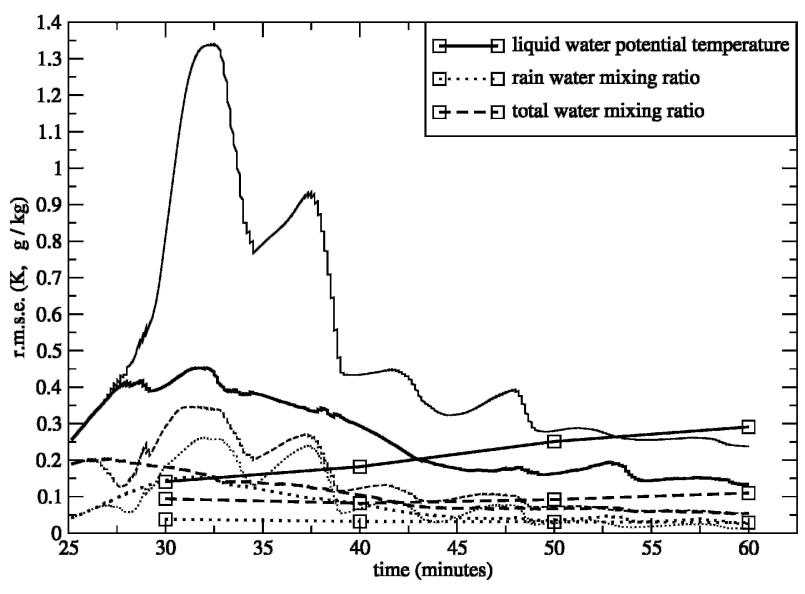

FIG. 13. As in Fig. 12 but for the liquid water potential temperature $\theta_{l}$, the rainwater content $q_{r}$, and the total water content $q_{t}$.

With further experiments, we have traced the major source of the difficulty to the assimilation of $q_{r}$ observations at early times. Without assimilation of $q_{r}$, the EnKF analyses are greatly improved (thick curves without squares in Figs. 12 and 13) and become better in quality than those produced when complete volumes of observations are available every $5 \mathrm{~min}$ (Fig. 2). Consistent with this result, Zhang et al. (2004) also assimilate only radial velocity, and also find that frequent assimilation of observations yields results comparable to those produced by assimilation every $5 \mathrm{~min}$.

Although we have not yet developed a definitive solution to this problem, the $q_{r}$ observations appear to degrade the analysis at early times as follows: Before the first observations are assimilated, the ensemble mean has disorganized vertical motion and too little $q_{r}$. The assimilation of radial-velocity observations begins to impose an updraft, and those members with the strongest updrafts tend to develop more $q_{r}$ in the next forecast. The $q_{r}$ observations, which are much larger than the ensemble-mean $q_{r}$, then produce further increases in $w$ owing to the positive correlation between $q_{r}$ and $w$. This results in much too strong an analyzed updraft after the first $3 \mathrm{~min}$ of assimilation. The fundamental problem is that the positive correlation between $w$ and $q_{r}$ at early times is an artifact of the ensemble initialization and is not representative of the errors in the ensemble mean (where errors in $w$ are positive while those in $q_{r}$ are negative).

\section{Summary}

This paper has compared a well-established 4DVAR implementation and a relatively new ensemble Kalman filter technique in the context of the assimilation of simulated radar data in a cloud-resolving model. By prescribing the observational error and neglecting the error of the forecast model, the interpretation of the results was easier in this first comparison of the two methods with a realistic atmospheric model at the convective scale.

Previous work with the EnKF at convective scales (Snyder and Zhang 2003; Zhang et al. 2004) did not explore the use of reflectivity observations. Rainwater content, as a surrogate for reflectivity, has been assimilated in the EnKF in this paper and shown to improve the analysis. [In an independent study, Tong and Xue (2005) also consider assimilation of reflectivity observations with the EnKF.] The improvement is most apparent after several assimilation cycles, at which point analyses that include rainwater observations have reduced rms error and smaller variability among realizations of the experiment. In the first cycles, however, rainwater observations in the EnKF can degrade the analysis if the prior ensemble mean is far from the true state (e.g., if it lacks the convective cell). The present study also employs a smooth spatial localization of the EnKF forecast covariances, which yields further improvements in the EnKF analyses relative to previous work in radar data assimilation. Here the "covariance relaxation" technique proposed by Zhang et al. (2004) did not improve the EnKF analyses. However, we speculate that this technique can be effective in the context of real data assimilation with the EnKF because it can compensate for the effect of model error.

In the simulations of supercells considered here, the model state has nearly $10^{6}$ degrees of freedom. The implementations of both schemes are therefore constrained by practical computational limits and require compromises relative to theoretically optimal algorithms. For example, the background in 4DVAR, as implemented here, is the horizontally uniform, environmental sounding rather than a forecast from the previous assimilation window, and the EnKF allows each observation to influence only state variables within a few kilometers of the observation location. Thus, our results necessarily reflect the specific implementation of each scheme as well as more fundamental properties of the underlying algorithms.

Both of the assimilation schemes generally perform well for the convective-scale flow considered in this study. Some differences were evident: 4DVAR has an advantage over the EnKF given observations over a limited period of, say, 10 min particularly for the wind components, while the EnKF typically produces better analyses than 4DVAR in later assimilation cycles.

The improvement of the EnKF analyses through suc- 
cessive cycles is clearly produced, at least in part, by the use of a prior forecast and estimates of its error covariance in the assimilation. The 4DVAR scheme employed here, in contrast, does not utilize forecasts from previous cycles (except as a first guess in the minimization), which, we believe, is one of the reasons why the 4DVAR analyses do not improve from one cycle to the next in most of the experiments except for the one starting at the mature stage of the storm. This is of course not a fundamental limitation of 4DVAR but rather a practical limitation of the scheme used here, which stems from the difficulty of modeling the background-error covariances at convective scales. Another possible reason why the 4DVAR analyses do not improve from one cycle to the next is that the convergence speed of the minimization in the 4DVAR scheme depends on the complexity of the cost function. For reasons that have been stated in section 3, we chose to terminate the minimization after 100 iterations for each 4DVAR run.

It is less clear why the EnKF analyses are inferior to those produced by 4DVAR when observations from only a limited time interval are used. One source of difficulty may be our specification of the initial ensemble for the EnKF. We have found (see also Snyder and Zhang 2003) that the performance of the EnKF, especially over the first few assimilation cycles, depends on the initial ensemble. Thus, there likely is potential for further improvements in the initial ensemble. Sampling error owing to the finite ensemble is another possibility, but our experiments shows little benefit from an increase of the ensemble size from 100 to $200 \mathrm{mem}$ bers. It is also difficult to quantify the benefits of the temporal smoothness constraint used in 4DVAR, which has no counterpart in the EnKF. At a more fundamental level, nonlinearity in the evolution of differences between members may also contribute to the differences in the 4DVAR and EnKF analyses.

Given the fact that the 4DVAR had a superior performance in the first cycle and the EnKF demonstrated a better error reduction with more cycles, a better analysis could be produced by combining the two schemes. One approach could be to use information from the EnKF in a hybrid scheme, although such a hybrid would require the development of new preconditioners for the 4DVAR minimization in order to remain computationally feasible for large problems. Another hybrid approach would be to use 4DVAR only over the first assimilation window, in order to provide an initial ensemble mean for the EnKF.

While comparisons of 4DVAR and the EnKF in this paper have been confined to an isolated convective cell, future multiscale numerical weather prediction models will resolve convective, mesoscale, and eventually global motions of the atmosphere, offering a great challenge for data assimilation. For 4DVAR, the difficulties lie in the fact that a 6-h assimilation window is a minimum required for the analysis of synoptic-scale motions. For a window of this length, however, multiple minima appear in the 4DVAR cost function because the evolution of errors in resolved meso- or smaller-scale features may be strongly nonlinear by the end of the window (Tanguay et al. 1995). It is not clear now how well the incremental approach can perform in this situation. For the EnKF, on the other hand, a large observation radius of influence will be required and problems of imbalance and degenerate ensemble covariances may affect the performance of the EnKF (Lorenc 2003). The relative performance of these two algorithms for problems with multiple scales will depend on how such issues are resolved in the future.

The comparison here has also considered only simulated observations and a perfect model. Another step in the comparison between 4DVAR and the EnKF will be in the context of the assimilation of real observations. This raises the issues of error in the forecast model and uncertainty in the specification of observational errors. Accounting properly for either of these are important research issues for both 4DVAR and the EnKF.

Acknowledgments. We are grateful to David Dowell who kindly shared with us his results during this project and to N. Andrew Crook who carefully commented on an earlier version of this manuscript. We also thank two anonymous reviewers for their comments leading to the clarification of several points in the paper. This research is supported at NCAR by the U.S. Weather Research Program (NCAR USWRP Grant I99-002) and by NSF Grant 0205655 .

\section{REFERENCES}

Anderson, J. L., 2001: An ensemble adjustment Kalman filter for data assimilation. Mon. Wea. Rev., 129, 2884-2903.

_ , and S. L. Anderson, 1999: A Monte Carlo implementation of the nonlinear filtering problem to produce ensemble assimilations and forecasts. Mon. Wea. Rev., 127, 2741-2758.

Bengtsson, T., C. Snyder, and D. Nychka, 2003: Toward a nonlinear ensemble filter for high-dimensional systems. J. Geophys. Res., 108, 8775, doi:10.1029/2002JD002900.

Cohn, S. E., 1997: An introduction to estimation theory. J. Meteor. Soc. Japan, 75, 257-288.

Courtier, P., and O. Talagrand, 1987: Variational assimilation of meteorological observations with the adjoint vorticity equation. Part II: Numerical results. Quart. J. Roy. Meteor. Soc., 113, 1329-1347.

Crook, N. A., and J. Sun, 2002: Assimilating radar, surface, and profiler data for the Sydney 2000 forecast demonstration project. J. Atmos. Oceanic Technol., 19, 888-898. 
Daley, R., 1991: Atmospheric Data Analysis. Cambridge University Press, $457 \mathrm{pp}$.

Dowell, D. C., F. Zhang, L. J. Wicker, C. Snyder, and N. A. Crook, 2004: Wind and temperature retrievals in the 17 May 1981 Arcadia, Oklahoma, supercell: Ensemble Kalman filter experiments. Mon. Wea. Rev., 132, 1982-2005.

Evensen, G., 1994: Sequential data assimilation with a nonlinear quasigeostrophic model using Monte Carlo methods to forecast error statistics. J. Geophys. Res., 99 (C5), 10 143-10 162.

Gaspari, G., and S. E. Cohn, 1999: Construction of correlation functions in two and three dimensions. Quart. J. Roy. Meteor. Soc., 125, 723-757.

Hamill, T. M., J. S. Whitaker, and C. Snyder, 2001: Distancedependent filtering of background error covariance estimates in an ensemble Kalman filter. Mon. Wea. Rev., 129, 27762790.

Hansen, J. A., 2002: Accounting for model error in ensemblebased state estimation and forecasting. Mon. Wea. Rev., 130, 2373-2391.

—- and L. A. Smith, 2001: Probabilistic noise reduction. Tellus, 53A, 585-598.

Houtekamer, P. L., and H. L. Mitchell, 1998: Data assimilation using an ensemble Kalman filter technique. Mon. Wea. Rev., 126, 796-811.

— atmospheric data assimilation. Mon. Wea. Rev., 129, 123-137.

Klinker, E., F. Rabier, G. Kelly, and J.-F. Mahfouf, 2000: The ECMWF operational implementation of four-dimensional variational assimilation. III: Experimental results and diagnostics with operational configuration. Quart. J. Roy. Meteor. Soc., 126, 1191-1215.

Le Dimet, F.-X., and O. Talagrand, 1986: Variational algorithms for analysis and assimilation of meteorological observations: Theoretical aspects. Tellus, 38A, 97-110.

Lewis, J. M., and J. C. Derber, 1985: The use of adjoint equations to solve a variational adjustment problem with advective constraints. Tellus, 37A, 309-322.

Lin, C.-L., T. Chai, and J. Sun, 2002: On the smoothness constraints for four-dimensional data assimilation. J. Comput. Phys., 181, 430-453.
Lorenc, A. C., 1986: Analysis methods for numerical weather prediction. Quart. J. Roy. Meteor. Soc., 112, 1177-1194.

_ 2003: The potential of the ensemble Kalman filter for NWP-A comparison with 4D-Var. Quart. J. Roy. Meteor. Soc., 129, 3183-3203.

Mitchell, H. L., and P. L. Houtekamer, 2000: An adaptive ensemble Kalman filter. Mon. Wea. Rev., 128, 416-433.

Reichle, R. H., D. B. McLaughlin, and D. Entekhabi, 2002: Hydrologic data assimilation with the ensemble Kalman filter. Mon. Wea. Rev., 130, 103-114.

Snyder, C., and F. Zhang, 2003: Assimilation of simulated Doppler radar observations with an ensemble Kalman filter. Mon. Wea. Rev., 131, 1663-1677.

Sun, J., and N. A. Crook, 1994: Wind and thermodynamic retrieval from single-Doppler measurements of a gust front observed during Phoenix II. Mon. Wea. Rev., 122, 1075-1091.

- and _ 1997: Dynamical and microphysical retrieval from Doppler radar observations using a cloud model and its adjoint. Part I: Model development and simulated data experiments. J. Atmos. Sci., 54, 1642-1661.

— and - 1998: Dynamical and microphysical retrieval from Doppler radar observations using a cloud model and its adjoint. Part II: Retrieval experiments of an observed Florida convective storm. J. Atmos. Sci., 55, 835-852.

—, and —, 2001: Real-time low-level wind and temperature analysis using single WSR-88D data. Wea. Forecasting, 16, $117-132$.

Tanguay, M., P. Bartello, and P. Gauthier, 1995: Four-dimensional data assimilation with a wide range of scales. Tellus, 47A, 974-997.

Tong, M., and M. Xue, 2005: Ensemble Kalman filter assimilation of Doppler radar data with a compressible nonhydrostatic model: OSS experiments. Mon. Wea. Rev., 133, 1789-1807.

Whitaker, J. S., and T. M. Hamill, 2002: Ensemble data assimilation without perturbed observations. Mon. Wea. Rev., 130, 1913-1924.

Zhang, F., C. Snyder, and J. Sun, 2004: Impacts of initial estimate and observation availability on convective-scale data assimilation with an ensemble Kalman filter. Mon. Wea. Rev., 132, $1238-1253$. 